\title{
Difference and efficacy of simplified motor scale compared to Glasgow coma scale
}

\author{
Tammam Mozher Aldarwish ${ }^{1 *}$, Mohammed Abdulaziz Alowaidhi ${ }^{2}$, Naish Abdullah Alghamdi ${ }^{3}$, \\ Ahmed Mohammed Al Hammad ${ }^{4}$, Mohammed Ibrahim Aljikhlib ${ }^{5}$, \\ Mohammad Mosa Alkhadhrawi ${ }^{6}$, Adil Ibrahim Hanbashi ${ }^{7}$, Ammar Yarmohammed Bakhsh ${ }^{8}$, \\ Fatimah Falah Al Matar', Amnah Saeed Al Saffar ${ }^{10}$, Ammar Zakaria Faloudah ${ }^{11}$
}

\author{
${ }^{1}$ Department of Emergency Medicine, King Fahad Genreal Hospital, Jeddah, Saudi Arabia \\ ${ }^{2}$ Department of Intensive Care Unit, Hera General Hospital, Mecca, Saudi Arabia \\ ${ }^{3}$ Department of Intensive Care Unit, Ahad Rufaidah General Hospital, Abha, Saudi Arabia \\ ${ }^{4}$ Department of Emergency Medicine, Qatif Central Hospital, Qatif, Saudi Arabia \\ ${ }^{5}$ Department of Internal Medicine, General Network of Healthcare Providers, Jeddah, Saudi Arabia \\ ${ }^{6}$ College of Medicine, Imam Abdulrahman Bin Faisal University, Dammam, Saudi Arabia \\ ${ }^{7}$ Department of Neurosurgery, King Fahad Central Hospital, Jazan, Saudi Arabia \\ ${ }^{8}$ Department of Emergency Medicine, King Faisal Hospital, Mecca, Saudi Arabia \\ ${ }^{9}$ College of Medicine, Medical University of Warsaw, Warsaw, Poland \\ ${ }^{10}$ Department of Emergency Medicine, Dammam Medical Complex, Dammam, Saudi Arabia \\ ${ }^{11}$ College of Medicine, Umm Al-Qura University, Mecca, Saudi Arabia
}

Received: 10 November 2021

Accepted: 25 November 2021

\section{*Correspondence:}

Dr. Tammam Mozher Aldarwish,

E-mail: tierdness55@gmail.com

Copyright: ( $)$ the author(s), publisher and licensee Medip Academy. This is an open-access article distributed under the terms of the Creative Commons Attribution Non-Commercial License, which permits unrestricted non-commercial use, distribution, and reproduction in any medium, provided the original work is properly cited.

\begin{abstract}
There have been many limitations reported with using the Glasgow coma scale (GCS), including complexity, and being difficult to apply among aphasic, intubated, and pediatric patients. Accordingly, many researchers exerted serious efforts to enhance and modify the scale to make it more applicable and easy to interpret in these settings. The simplified motor score (SMS) was reported in the literature in 2012 for the assessment of patients with coma in different traumatic and non-traumatic settings. In the present study, we have discussed the findings of previous studies in the literature that compared the efficacy between the SMS and GCS in the assessment of patients with traumatic brain injuries within the emergency department and out-patient settings. Our results indicate the efficacy of the SMS is similar to that of the GCS score in predicting the different outcomes, including functional performance, need to perform tracheal intubation and hospital admission. Nevertheless, evidence regarding the prediction of mortality seems to be inconsistent across the different investigations. However, the differences between the two scores is not remarkable among these studies, indicating that the SMS is an efficacious tool in this regard within an acceptable test performance results. Furthermore, the SMS score can be easily applied within these without performing complex approaches, which makes it more advantageous than the GCS. However, this evidence is based on a limited number of investigations, and more studies are required.
\end{abstract}

Keywords: GCS, SMS, Coma, Emergency 


\section{INTRODUCTION}

Since the Glasgow coma scale (GCS) was first introduced and reported in the literature, it has been widely used until the current days within the different healthcare settings for evaluation of impaired consciousness and assessment of coma among patients suffering from stroke, traumatic brain injuries, and other conditions at the emergency department. ${ }^{1-4}$ However, there have been many limitations reported with using this scale, including complexity, and being difficult to apply among aphasic, intubated, and pediatric patients. Accordingly, many researchers exerted serious efforts to enhance and modify the scale to make it more applicable as well as easy to the interpret in these settings.

The simplified motor score (SMS) was reported in the literature in 2012 for the assessment of patients with coma in different traumatic and non-traumatic settings. ${ }^{5}$ Many studies were then conducted to compare the efficacy of the SMS with the GCS and furtherly validate it. In the present literature review, we aim to compare the efficacy of the SMS with GCS based on evidence from studies in the literature.

\section{LITERATURE REVIEW}

This literature review is based on an extensive literature search in Medline, Cochrane, and EMBASE databases which was performed on $26^{\text {th }}$ October 2021 using the medical subject headings (MeSH) or a combination of all possible related terms, according to the database. To avoid missing potential studies, a further manual search for papers was done through Google Scholar while the reference lists of the initially included papers. Papers discussing difference and efficacy of SMS compared GCS were screened for useful information. No limitations were posed on date, language, age of participants, or publication type.

\section{DISCUSSION}

Many studies have investigated such outcomes, and we tried to collect all the potential evidence that can help us validated these outcomes and formulate a proper discussion. Following the previous investigation by Tuhrim et al that was conducted in 1988 and showed that among $92 \%$ of the included patients, the GCS was significantly able to classify them into dead and alive based on their clinical data, the scale has been met with great acceptance across the different settings, especially within the emergency department. ${ }^{6}$ The scale was able to predict death after 30 days from intracranial hemorrhage took place. In the same context, another retrospective investigation that was conducted by $\mathrm{Fu}$ et al and included 1268 patients that primarily suffered from intracranial hemorrhage, reported that in-hospital mortality after intracranial hemorrhage and severity on admission was significantly associated with the GCS. ${ }^{7}$ Other scores were also developed following these studies for further validation and adequately predicting severe conditions to provide the necessary care for these patients and enhance their outcomes. Another previous investigation suggested that the prognosis of intracranial hemorrhage can be significantly assessed using the GCS. It has been shown that there was a significant association between the GCS and the clinical outcomes of patients with intracranial hemorrhage, and a previous study reported that the area under the curve for predicting 30-day mortality following hospital admission secondary to intracranial hemorrhage was 0.871 [ $95 \%$ confidence interval $(\mathrm{CI}), 0.817$ to 0.913 , $\mathrm{p}<0.001] .{ }^{8}$ These results were also consistent with the findings of a previous study by Jamil et al that concluded that the ability of the GCS to predict mortality after 30 days from admission secondary to intracranial hemorrhage was statistically significant in both the uniand multi-variate analysis. ${ }^{9}$ Furthermore, poor outcomes of intracranial hemorrhage were also reported to be significantly correlated with the GCS. ${ }^{8}$ This has been furtherly indicated in a previous investigation by Cho et al that concluded that 30-day mortality in their 200 patients with intracranial hemorrhage was significantly associated with the GCS. Similar findings were also reported in another investigation by Grmec and Gašparovic. $^{10,11}$

The GCS is currently being used in different settings for the assessment of patients with coma due to different etiologies, including traumatic brain injury, and the efficacy has been validated by many researchers and widely accepted across clinical settings. However, the scale has been reported to have some limitations. For instance, evidence shows that the reliability and accuracy of assessment with the GCS can be significantly impacted by the experience and knowledge of the tester. ${ }^{8,12}$ This has been furtherly indicated in another investigation by Holdgate et al which showed that when measuring GCS within the emergency department, there was a huge variability between the nurses and physicians. ${ }^{13}$ Other studies also reported that although there is wide acceptance of GCS in these settings and significant efficacy, some concerns should still be considered because some variations were reported between many patients in $\geq 1$ point of the GCS. It has been furtherly reported in a previous investigation by Buechler et al that the validity of the GCS can be significantly impacted by some variations that were noticed during the assessment of sedated and intubated patients, which affected the reliability of the scale in these settings. ${ }^{14}$ Therefore, it has been demonstrated that the validity of predicting coma outcomes in these patients can be significantly impacted. A radical suggestion was also found in the literature that the GCS should not be used anymore in these settings based on these documented limitations. ${ }^{15}$ However, this suggestion was not widely accepted among physicians and researchers based on the long history of favorable outcomes with the GCS. Accordingly, many researchers aimed to develop novel approaches that can overcome the limitations of the GCS and enhance the associated outcomes, and therefore, many other scales were reported 
and validated. Among the different scales, SMS has been reported with great results and promising predicting abilities, and many investigations have compared it with the GCS in the settings of traumatic brain injury.

More and more evidence can be found in the literature about the efficacy and validity of the SMS, among studies that compare it with the GCS, for the determination of the prognosis for patients with severe stroke and traumatic brain injury.,16-18 A previous investigation that included patients with intracranial hemorrhage reported that the accuracy of SMS was significantly similar to the efficacy of GCS in predicting poor outcomes in these patients. Another study also demonstrated that predicting 30-day mortality among patients with intracranial hemorrhage was significantly achieved by $\mathrm{SMS} \leq 0$, and GCS $\leq 5$, which indicates that the SMS is significant as efficacious as the GCS in predicting mortality in these patients. ${ }^{19}$ However, it should be noted that test performance is significantly lower than the estimated performance for the GCS in properly predicting mortality among patients with intracranial hemorrhage, as estimated by the AUC values for both modalities. The clinical outcomes of patients with intracranial hemorrhage were also significantly correlated with the scores of SMS. The authors of this study also conducted a correlation analysis between GCS and SMS and APACHE II scores in assessing coma in their patients. It has been demonstrated that both of these scales were significantly correlated with the APACHE II score, indicating the validity and reliability of SMS in predicting clinical outcomes of patients with intracranial hemorrhage. It has been furtherly shown that the different classifications of the SMS were significantly variable with the different scores, including GCS and APACHE II scores. ${ }^{8}$ Evidence shows that there might be a direct association between SMS and coma outcomes (for instance, higher SMS scores indicate better outcomes). This indicates that the SMS can be used in the clinical settings, having similar outcomes to the GCS. However, favoring SMS over the GCS is still an area of concern, and some researchers even suggested that it can be reliably used, being more practical and easier than the GCS which might difficult to be obtained within certain circumstances in the emergency settings. ${ }^{8}$

A previous meta-analysis reported that the predictive ability of the SMS was good and was comparable to the efficacy of the GCS in predicting neurosurgical intervention, clinically-related brain injury, and emergency tracheal intubation for patients suffering from traumatic brain injuries. On the other hand, it has been reported that mortality was better predicted by the GCS score. Nevertheless, it should be noted that the difference between SMS and GCS score in the assessment of this outcome was not statistically significant, and it has been reported that such evidence needs to be furtherly demonstrated by other investigations. ${ }^{4}$ These outcomes were reported for patients within the out-of-hospital and emergency settings. However, it should be noted that the authors of this meta-analysis reported that heterogeneity was significant among the included studies, which might affect the findings of this investigation. Furthermore, patients with less severe forms of injury, including concussions, unspecified head injuries, and fractures without intracranial effect were usually excluded from the included studies in this meta-analysis, which might have also impacted the results as these patients represent the majority of patients with intracranial hemorrhage.

The domains of the SMS are usually based on the motor component of the GCS across the different investigations in the literature. As the verbal score is not provided in the GCS score, it has been reported that the SMS score has an advantage over the GCS in this context, being able to assess non-verbal and intubated patients. Inter-rater reliability was also reported to be significantly lowest in the verbal component among other components of the score. Compared to the verbal and motor components, evidence also shows that the predictive value for the eye component is also weak when assessed by the GCS score. ${ }^{20}$ Evidence indicates that the predictive value of the motor component of the GCS score is statistically significant and equal to the total score. Nonetheless, it should be noted that not sufficient data is available regarding the assessment of the different outcomes for patients with traumatic brain injuries. ${ }^{21-24}$ In fact, the 6point motor GCS score was similar to the 3-point SMS scale in the overall test performance. This has been validated in the predictive ability of these scores in the settings of mortality, clinically significant brain injury, neurosurgical interventions, and emergency tracheal intubation. ${ }^{4}$ These findings indicate that the SMS scale is as efficacious as the GCS score. Furthermore, the SMS score is not complex, easy to use, faster to calculate, and more simple than the GCS score, which might make it more favorable to use in the settings of assessment of traumatic brain injury. Studies also demonstrated that the full outline of unresponsiveness (FOUR) score has been reported as an efficacious alternative to the GCS scale. Nevertheless, it has been shown that the tool has been rated with limited inter reliability, requires increased time to be adequately used, and is complicated to used even by trained personnel, and therefore, it might be difficult to apply within the emergency department for prediction of the different outcomes. ${ }^{20,25,26}$ On the other hand, the SMS score is easy to apply and concise as compared to the GCS score, which has been reported to be difficult to remember and cannot be easily applied. ${ }^{27-30}$ Accordingly, the SMS can add a significant value to the assessment and prognosis of traumatic brain injuries within the outpatient and emergency department settings with comparable test performance to the GCS score. However, the current evidence seems to lack data from observational investigations, and therefore, further studies are needed to the adequately validate the current findings by prospective data. In addition, these studies are encouraged to overcome the current limitations of the different investigations in the literature for the better validation. 


\section{CONCLUSION}

The efficacy of the SMS is similar to that of the GCS score in predicting the different outcomes, including functional performance, need to perform tracheal intubation and hospital admission. However, evidence regarding the prediction of mortality seems to be inconsistent across the different investigations. Nevertheless, the differences between the two scores is not remarkable among these studies, indicating that the SMS is an efficacious tool in this regard within an acceptable test performance results. Furthermore, the SMS score can be easily applied within these settings without performing complex approaches, which makes it more advantageous than the GCS. However, this evidence is based on a limited number of investigations, and more studies are required.

Funding: No funding sources Conflict of interest: None declared

Ethical approval: Not required

\section{REFERENCES}

1. Hifumi T, Kuroda Y, Kawakita K. Effect of Admission Glasgow Coma Scale Motor Score on Neurological Outcome in Out-of-Hospital Cardiac Arrest Patients Receiving Therapeutic Hypothermia. Circulation j. 2015;79(10):2201-8.

2. Handschu R, Haslbeck M, Hartmann A. Mortality prediction in critical care for acute stroke: Severity of illness-score or coma-scale? J neurol. 2005;252(10):1249-54.

3. McNett M. A review of the predictive ability of Glasgow Coma Scale scores in head-injured patients. J neurosci. 2007;39(2):68-75.

4. Singh B, Murad MH, Prokop LJ. Meta-analysis of Glasgow coma scale and simplified motor score in predicting traumatic brain injury outcomes. Brain injury. 2013;27(3):293-300.

5. Caterino JM, Raubenolt A. The prehospital simplified motor score is as accurate as the prehospital Glasgow coma scale: analysis of a statewide trauma registry. Emergency med j. 2012;29(6):492-6.

6. Tuhrim S, Dambrosia JM, Price TR. Prediction of intracerebral hemorrhage survival. Ann neurol. 1988;24(2):258-63.

7. Fu X, Wong KS, Wei JW. Factors associated with severity on admission and in-hospital mortality after primary intracerebral hemorrhage in China. Int $\mathrm{j}$. 2013;8(2):73-9.

8. Jina L, Yangb P, Zhang Y. Prediction Values of the Simplified Motor Score and the Glasgow Coma Scale Motor Component for the Clinical Outcomes of Intracranial Hemorrhage. 2021.

9. Jamil SA, Khan AS, Akturk Z. Predictors of outcome for non-traumatic intracerebral hemorrhage. Neurosciences (Riyadh, Saudi Arabia). 2008;13(3):263-7.
10. Cho DY, Wang YC. Comparison of the APACHE III, APACHE II and Glasgow Coma Scale in acute head injury for prediction of mortality and functional outcome. Intensive care med. 1997;23(1):77-84.

11. Grmec S, Gasparovic V. Comparison of APACHE II, MEES and Glasgow Coma Scale in patients with nontraumatic coma for prediction of mortality. Acute Physiology and Chronic Health Evaluation. Mainz Emergency Evaluation System. Cri care. 2001;5(1):19-23.

12. Rowley G, Fielding K. Reliability and accuracy of the Glasgow Coma Scale with experienced and inexperienced users. Lancet. 1991;337(8740):535-8.

13. Holdgate A, Ching N, Angonese L. Variability in agreement between physicians and nurses when measuring the Glasgow Coma Scale in the emergency department limits its clinical usefulness. Emergency med Australasia. 2006;18(4):379-84.

14. Buechler CM, Blostein PA, Koestner A, Hurt K, Schaars M, McKernan J. Variation among trauma centers' calculation of Glasgow Coma Scale score: results of a national survey. $\mathbf{J}$ Trauma. 1998;45(3):429-32.

15. Green SM. Cheerio, laddie! Bidding farewell to the Glasgow Coma Scale. Ann emergency med. 2011;58(5):427-30.

16. Buitendag JJP, Ras A, Kong VY. Validation of the Simplified Motor Score in patients with traumatic brain injury at a major trauma centre in South Africa. South Afri med j. 2018;108(2):90-3.

17. Wang JP, Su YY, Liu YF, Liu G, Fan LL, Gao DQ. Study of Simplified Coma Scales: Acute Stroke Patients with Tracheal Intubation. Chin med j. 2018;131(18):2152-7.

18. Gill M, Windemuth R, Steele R, Green SM. A comparison of the Glasgow Coma Scale score to simplified alternative scores for the prediction of traumatic brain injury outcomes. Ann emergency med. 2005;45(1):37-42.

19. Ting HW, Chen MS, Hsieh YC, Chan CL. Good mortality prediction by Glasgow Coma Scale for neurosurgical patients. J Chin Med Asso. 2010;73(3):139-43.

20. Gill MR, Reiley DG, Green SM. Interrater reliability of Glasgow Coma Scale scores in the emergency department. A emergency med. 2004;43(2):215-23.

21. Healey C, Osler TM, Rogers FB. Improving the Glasgow Coma Scale score: motor score alone is a better predictor. J Trauma. 2003;54(4):671-8.

22. Al-Salamah MA, McDowell I, Stiell IG. Initial emergency department trauma scores from the OPALS study: the case for the motor score in blunt trauma. Academic emergency med. 2004;11(8):83442.

23. Ross SE, Leipold C, Terregino C, O'Malley KF. Efficacy of the motor component of the Glasgow Coma Scale in trauma triage. J Trauma. 1998;45(1):42-4.

24. Meredith W, Rutledge R, Hansen AR. Field triage of trauma patients based upon the ability to follow 
commands: a study in 29,573 injured patients. J Trauma. 1995;38(1):129-35.

25. Wijdicks EF, Bamlet WR, Maramattom BV, Manno EM, McClelland RL. Validation of a new coma scale: The FOUR score. Anna neurol. 2005;58(4):585-93.

26. Fischer M, Rüegg S, Czaplinski A. Inter-rater reliability of the Full Outline of UnResponsiveness score and the Glasgow Coma Scale in critically ill patients: a prospective observational study. Critical care. 2010;14(2):R64.

27. Van de Voorde P, Sabbe M, Rizopoulos D. Assessing the level of consciousness in children: a plea for the Glasgow Coma Motor subscore. Resuscitation. 2008;76(2):175-9.
28. Riechers RG, 2nd, Ramage A, Brown W. Physician knowledge of the Glasgow Coma Scale. J neurotrauma. 2005;22(11):1327-34.

29. McNarry AF, Goldhill DR. Simple bedside assessment of level of consciousness: comparison of two simple assessment scales with the Glasgow Coma scale. Anaesthesia. 2004;59(1):34-7.

30. Jennett B. The Glasgow Coma Scale: History and current practice. Trauma. 2002;4(2):91-103.

Cite this article as: Aldarwish TM, Alowaidhi MA, Alghamdi NA, Al Hammad AM, Aljikhlib MI, Alkhadhrawi MM et al. Difference and efficacy of simplified motor scale compared to Glasgow coma scale. Int J Community Med Public Health 2022;9:327-31. 